\title{
X-ray Photoelectron Spectroscopic Analysis of Si Nanoclusters in $\mathrm{SiO}_{2}$ Matrix
}

\author{
Aykutlu Dane, ${ }^{\dagger}$ U. Korcan Demirok,,${ }^{\ddagger}$ Atilla Aydinli, ${ }^{\dagger}$ and Sefik Suzer*, \\ Bilkent University, Physics Department, 06800 Ankara, Turkey, and Bilkent University, Chemistry Department, \\ 06800 Ankara, Turkey
}

Received: August 15, 2005; In Final Form: November 24, 2005

\begin{abstract}
We investigated silicon nanoclusters $\mathrm{Si}(\mathrm{nc})$ in a $\mathrm{SiO}_{2}$ matrix prepared by the plasma-enhanced chemical vapor deposition technique, using X-ray photoelectron spectroscopy (XPS) with external voltage stimuli in both static and pulsed modes. This method enables us to induce an additional charging shift of $0.8 \mathrm{eV}$ between the $\mathrm{Si} 2 \mathrm{p}$ peaks of the oxide and the underlying silicon, both in static and time-resolved modes, for a silicon sample containing a $6 \mathrm{~nm}$ oxide layer. In the case of the sample containing silicon nanoclusters, both Si2p peaks of $\mathrm{Si}(\mathrm{nc})$ and host $\mathrm{SiO}_{2}$ undergo a charging shift that is 1 order of magnitude larger $(>15 \mathrm{eV})$, with no measurable difference between them (i.e., no differential charging between the silicon nanoclusters and the oxide matrix could be detected). By use of a measured Auger parameter, we estimate the relaxation energy of the $\mathrm{Si}(\mathrm{nc})$ in the $\mathrm{SiO}_{2}$ matrix as $-0.4 \mathrm{eV}$, which yields a $-0.6 \mathrm{eV}$ shift in the binding energy of the $\mathrm{Si}(\mathrm{nc})$ with respect to that of bulk $\mathrm{Si}$ in the opposite direction of the expected quantum size effect. This must be related to the residual differential charging between the silicon nanoclusters and the oxide host. Therefore, differential charging is still the biggest obstacle for extracting size-dependent binding energy shifts with XPS when one uses the oxide peak as the reference.
\end{abstract}

\section{Introduction}

Interest in silicon nanoclusters, $\mathrm{Si}(\mathrm{nc})$, is ever increasing due to their potential for incorporation into micro- and/or optoelectronic devices and systems. ${ }^{1-9}$ Several physical/chemical techniques have been developed for their preparation; among these ion implantation and plasma-enhanced chemical vapor deposition (PECVD) are the most successful techniques. ${ }^{10,11}$ In addition to X-ray diffraction (XRD), scanning electron microscopy and transmission electron microscopy (SEM and TEM), and tunneling techniques such as scanning tunneling microscopy (STM) and scanning capacitance microscopy (SCM) have widely been employed for optical, electrical, and structural characterization of these nanoclusters. ${ }^{12,13}$ For chemical characterization, spectroscopic techniques such as IR, Raman, UV-Vis-NIR, X-ray absorption, and X-ray photoemission have been utilized. ${ }^{14,15}$ $\mathrm{X}$-ray photoelectron spectroscopy (XPS) is especially powerful due to the perfect match of its probe length with the size of these nanoclusters, and several articles have been published characterizing the silicon nanoclusters, mostly using the chemical shifts to derive information about the nature and the charge state of $\mathrm{Si}$ in the nanoclusters and/or the effect of the surrounding matrix on these clusters. ${ }^{14-21}$

Since silicon nanoclusters are usually embedded in an insulating matrix, charging is an experimental obstacle to accurate separation of the various chemical/physical parameters contributing to the derived chemical shifts. ${ }^{22}$ In an attempt to determine the quantum size effect of the silicon nanoclusters embedded in a $\mathrm{SiO}_{2}$ matrix, Chen et al. recently reported the variations observed in the chemical shifts of the five oxidation states $\mathrm{Si}^{n+}(n=0,1,2,3$, and 4$)$ during annealing of the nanoclusters, after correcting for charging by using $\mathrm{C} 1 \mathrm{~s}$ peak

\footnotetext{
* To whom correspondence should be addressed. E-mail: suzer@ fen.bilkent.edu.tr.

Bilkent University, Physics Department.

$\doteqdot$ Bilkent University, Chemistry Department.
}

(resulting from the contamination on the oxide surface). ${ }^{23}$ Although this correction brought down the observed binding energy of the $\mathrm{Si}^{4+}$ to that of bulk $\mathrm{SiO}_{2}$, there was no internal reference that could be used for the $\mathrm{Si}(\mathrm{nc})$. Normally, smaller clusters are expected to exhibit a positive binding energy shift from their bulk values because of their smaller size. However, Chen et al.'s finding for the Si2p binding energy suggested ca. $\mathrm{a}-0.6 \mathrm{eV}$ shift for the $3.0 \mathrm{~nm} \mathrm{Si(nc)} \mathrm{in} \mathrm{the} \mathrm{opposite} \mathrm{direction,}$ which they attributed to differential charging between the oxide host and the $\mathrm{Si}(\mathrm{nc})$.

Although accurate referencing for the $\mathrm{Si}(\mathrm{nc})$ seems hopeless, one practical experimental approach-using controlled chargingcan offer some help in this regard. As we reported recently, one can control the charging of various surface structures by the application of voltage stimuli in static and/or pulsed modes to the sample rod while recording XPS spectra. ${ }^{24-29}$ In this contribution, we extend our application to samples containing silicon nanoclusters $\mathrm{Si}(\mathrm{nc})$ in ca. $300 \mathrm{~nm}$ thin $\mathrm{SiO}_{2}$ films prepared using the PECVD technique; we are attempting to offer further experimental data for elucidating various chemical/ physical shifts of small nanoparticles measured by XPS.

\section{Experimental Section}

The silicon-rich oxide films were grown in a PECVD reactor (PlasmaLab 8510C) on precleaned (001) Si substrates using 180 sccm $\mathrm{SiH}_{4}\left(2 \%\right.$ in $\left.\mathrm{N}_{2}\right)$ and $22 \mathrm{sccm} \mathrm{NO} \mathrm{NO}_{2}$ as precursor gases, at a sample temperature of $350{ }^{\circ} \mathrm{C}$, a process pressure of 1000 mTorr under an applied RF power of $10 \mathrm{~W}$. Different film thicknesses were obtained by growing for calibrated times. Samples were then annealed in a $\mathrm{N}_{2}$ atmosphere in a quartz oven at temperatures of $900-1200{ }^{\circ} \mathrm{C}$ for $2 \mathrm{~h}$. The samples were loaded and unloaded rapidly, resulting in ramp times of about $1 \mathrm{~min}$. Control samples were prepared in the same way except that $\mathrm{NO}_{2}$ flow rate was $180 \mathrm{sccm}$ resulting in near stoichiometric $\mathrm{SiO}_{2}$ films. Thickness and the refractive indices 


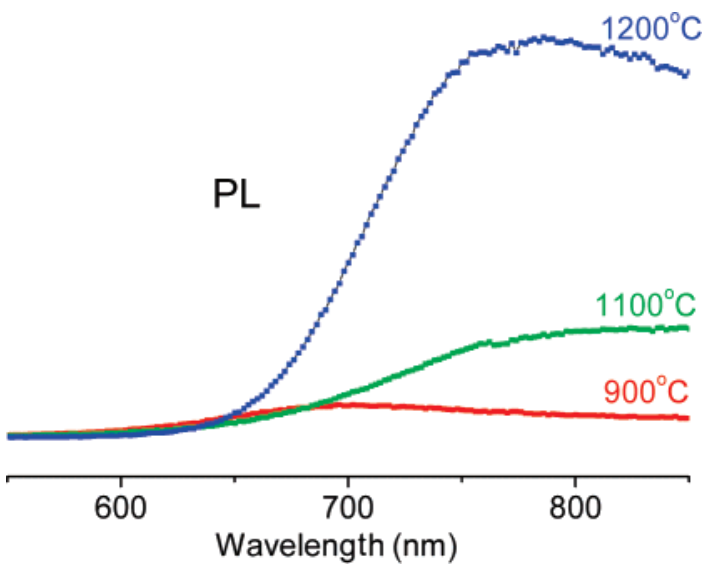

Figure 1. Relative photoluminescence intensity of the $\mathrm{Si}(\mathrm{nc})$ in a ca. $300 \mathrm{~nm} \mathrm{SiO}{ }_{2}$ matrix sample annealed at three different temperatures using a 514.5-nm line of an $\mathrm{Ar}^{+}$laser.

of all samples were measured using a Rudolph Elipsometer at $632 \mathrm{~nm}$, and the thickness values were further verified by using a stylus profilometer. Photoluminescence measurements were performed using the 514.5-nm line of ${\mathrm{an} \mathrm{Ar}^{+}}^{+}$laser. Spectra were collected through a monochromator (Jobin Yvon U1000) using a PMT with a GaAs photocathode and the necessary photoncounting electronics.

A Kratos ES300 electron spectrometer with $\mathrm{Mg} \mathrm{K} \alpha$ X-rays (nonmonochromatic) is used for XPS measurements. The X-ray tube also emits broad and more energetic bremsttrahlung X-rays, which lead to production of characteristic Auger peaks (albeit with reduced intensity). For Si the more intense components of the $\mathrm{Si}_{\mathrm{KLL}}$ are observed around $1600 \mathrm{eV}$. A typical sample is a ca. $2 \mathrm{~mm}$ thick wafer with dimensions of $4 \times 8 \mathrm{~mm}$; it is tied to the sample rod and the voltage stress (direct current (dc) or pulsed) is applied externally to the sample rod. A filament is placed within $5 \mathrm{~cm}$ of the sample to supply extra low energy electrons. ${ }^{26}$ For time-resolved measurements, the bias is stepped and pulsed. During each pulse, 200 measurements with 10-ms resolution are recorded; the voltage is stepped and pulsed for the next 200 measurements until a region is completed. Several scans are accumulated for signal averaging. The result is a collection of 200 time-resolved spectra. ${ }^{27,29}$

\section{Results and Discussion}

Figure 1 shows the photoluminescence of the sample which has ca. $300 \mathrm{~nm}$ thick oxide layer (containing also the silicon nanoparticles) after annealing at 900,1100 , and $1200{ }^{\circ} \mathrm{C}$; this displays an increasing PL intensity with temperature. Although it is accepted that defects also contribute to PL, defect-related PL peaks in oxides and nitrides are known to generally decrease with increasing annealing time and temperature. However, Si(nc) peaks are known to enhance in magnitude and shift toward red due to their increased size. ${ }^{1-4}$ Moreover, defect related PL is generally observed between 350 and $600 \mathrm{~nm}$ and does not red shift with annealing. On the basis of the observed enhancement and red shift of the PL with increasing annealing time and temperature, we conclude that our samples contain silicon nanocrystals. ${ }^{3,5}$ Another piece of evidence for the presence of nanocrystals comes from capacitance-voltage (CV) measurements. Accordingly, MOS capacitors with bare oxide do not show any hysteresis in the CV curves, whereas capacitors with $\mathrm{Si}(\mathrm{nc})$ show hysteresis that depends on the nanocrystal density (adjusted by annealing temperature), ${ }^{6}$ which is also the case for the samples used in this work.

In Figure 2 we show spectra corresponding to the $\mathrm{Si}_{\mathrm{KLL}}$ Auger

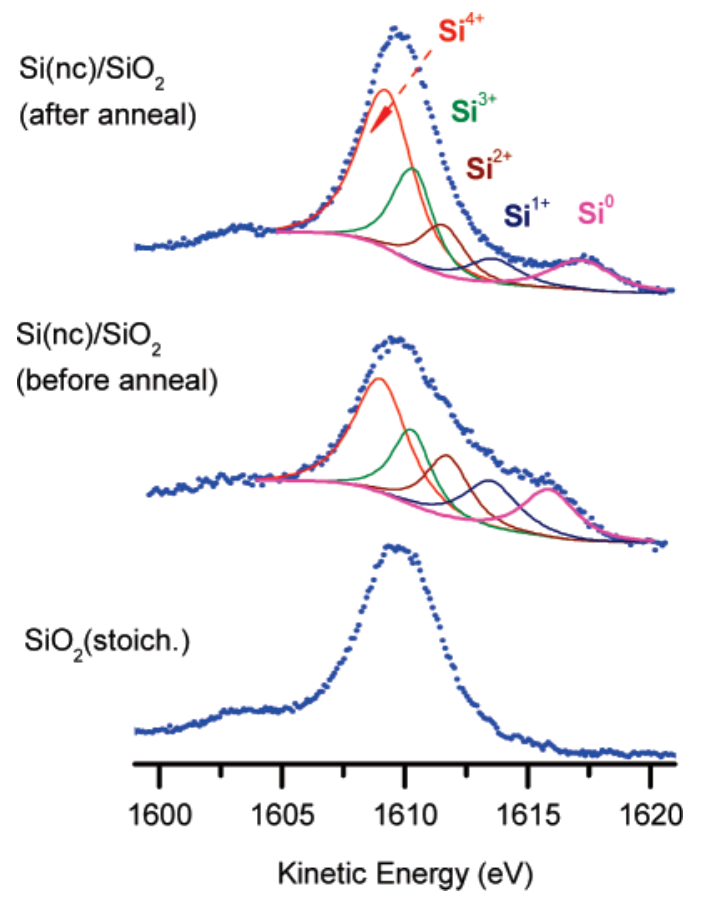

Figure 2. Part of the XPS spectrum corresponding to the $\mathrm{Si}_{\mathrm{KLL}}$ Auger region of the sample as in Figure 1 before and after annealing at 1100 ${ }^{\circ} \mathrm{C}$ together with a near stoichiometric $\mathrm{SiO}_{2}$ sample for comparison.

peaks of the sample before and after annealing at $1100{ }^{\circ} \mathrm{C}$. Spectrum of a near-stoichiometric $\mathrm{SiO}_{2}$ sample is also reproduced for comparison. Since the oxide layer is ca. $300 \mathrm{~nm}$ for all these samples, the peak corresponding to the silicon substrate cannot be observed. As can be gathered from the figure, for the sample containing $\mathrm{S}(\mathrm{nc})$ additional features at high kinetic energy side are present, which become better separated after annealing. We chose the Auger region to reflect our observations since it is well known that the chemical shifts between various oxidation states are more pronounced (roughly twice) when compared to Si2p photopeaks. ${ }^{30}$ Accordingly, we fit the composite peak to five different components corresponding to the $0,+1,+2,+3$, and +4 charge states of $\mathrm{Si}$, using the XPSPEAK95 Version 2.0. ${ }^{31}$ Upon annealing two spectral features emerge, (i) all the suboxides and the $\mathrm{Si}^{0}$ peaks lose their intensities due to enhanced oxidation during annealing and (ii) the binding energy difference between the $\mathrm{Si}^{0}(\mathrm{nc})$ and the $\mathrm{Si}^{4+}$ peaks increases, due most probably to better phase separation and, more importantly, to enhanced differential charging (see the discussion below). As shown in Figure 3, the $\mathrm{Si} 2 \mathrm{p}$ region is also fitted to 5 components yielding a difference of $4.3 \pm 0.1 \mathrm{eV}$ between the $\mathrm{Si}^{0}$ (nc) and the $\mathrm{Si}^{4+}$ peaks. Taking the bulk value of the $\mathrm{Si} 2 \mathrm{p}$ binding energy of the $\mathrm{SiO}_{2}$ as 103.4 , we obtain $99.1 \mathrm{eV}$ for the $\mathrm{Si} 2 \mathrm{p}$ binding energy of the $\mathrm{Si}(\mathrm{nc})$ in this $\mathrm{SiO}_{2}$ matrix, which is $-0.2 \pm 0.1 \mathrm{eV}$ lower than the bulk value of $\mathrm{Si}(99.3 \mathrm{eV})$.

Three physicochemical factors contribute to the obtained binding energy shift: (i) quantum size effect which is expected to give a positive shift, (ii) the relaxation effect of the matrix, defined as the response of the matrix to the photohole created, this too is expected to give a positive contribution in the case of the silicon oxide matrix, and (iii) to the differential charging between the $\mathrm{Si}(\mathrm{nc})$ and the oxide host. The Auger parameter (AP), $\alpha$, which is defined as the sum of the binding energy of the $\mathrm{Si} 2 \mathrm{p}$ peak and the kinetic energy of the corresponding $\mathrm{Si}_{\mathrm{KLL}}$ Auger peak, is another experimental parameter reflecting the effects of the particle size, and/or the matrix. In addition, the $\mathrm{AP}$ is known to be free from charging effects. ${ }^{30,32-34}$ Our measured $\mathrm{AP}$ for the $\mathrm{Si}(\mathrm{nc})$ in the $\mathrm{SiO}_{2}$ matrix is $1715.1 \pm 0.1$ 


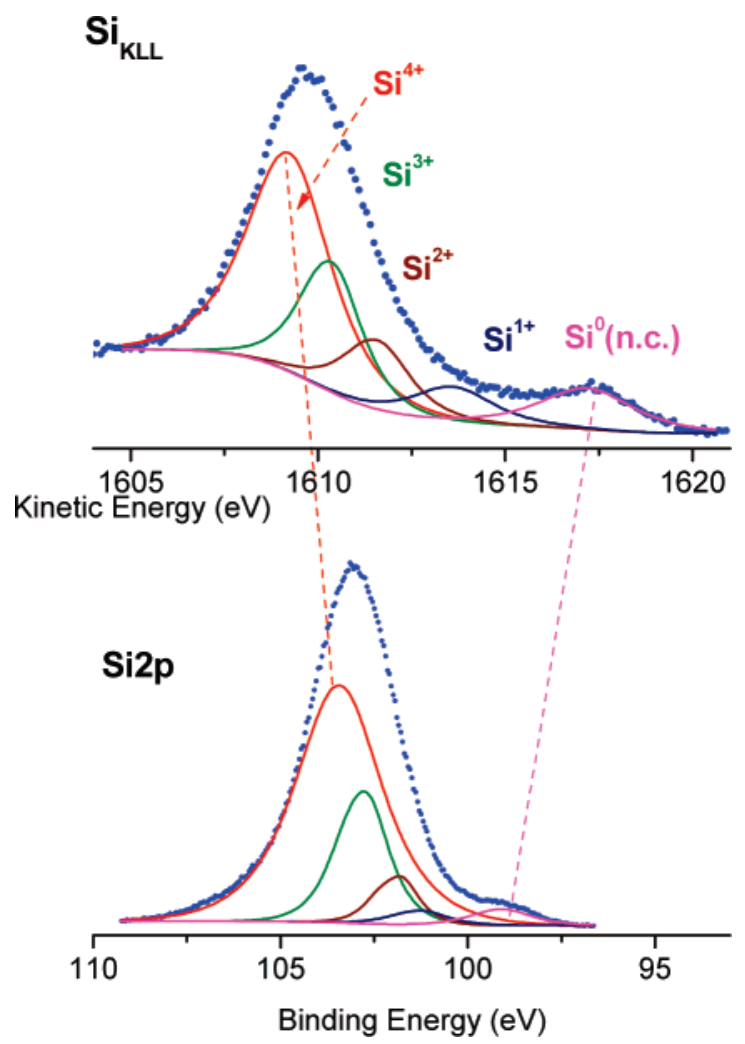

Figure 3. Part of the XPS spectrum of the annealed sample corresponding to $\mathrm{Si} 2 \mathrm{p}$ and the $\mathrm{Si}_{\mathrm{KLL}}$ Auger regions. Both regions are curvefitted to 5 components corresponding to $\mathrm{Si}^{n+}(n=4,3,2,1$, and 0$)$.

$\mathrm{eV}$, slightly lower than the value of the bulk $\mathrm{Si}(1715.9 \mathrm{eV})$. The difference $(\Delta \alpha=-0.8 \mathrm{eV})$ is within the expected magnitude and direction, since it is well established that the less electronically conducting matrix decreases the AP; for example, the corresponding value for $\mathrm{Al}$ on $\mathrm{SiO}_{2}$ is $-1.10 \mathrm{eV}$ smaller than that on $\mathrm{Si}$, which is a further $-0.60 \mathrm{eV}$ smaller than that on $\mathrm{Cu}$, due to the matrix relaxation effects. ${ }^{34}$ Since our experimental finding reveals the presence of silicon suboxides, there must be few layers of these suboxides in the immediate vicinity of the $\mathrm{Si}(\mathrm{nc})$; these are expected to provide a smaller relaxation shift than the full oxide, in agreement with our findings. The contribution of the relaxation effect on binding energy shift can be estimated using the simple approximation introduced by Wagner and Thomas ${ }^{30,32}$

$$
\Delta \mathrm{BE}[\mathrm{Si}(\mathrm{nc})-\mathrm{Si}(\text { bulk })]=-0.2 \mathrm{eV}=\Delta \epsilon-\Delta \mathrm{RE}
$$

and

$$
\Delta \alpha=2 \Delta \mathrm{RE}=-0.8 \pm 0.1 \mathrm{eV}
$$

where, $\Delta \mathrm{BE}, \Delta \epsilon$, and $\Delta \mathrm{RE}$ are, respectively, the binding energy difference, the true chemical shift, and the relaxation energy difference between the $\mathrm{Si}(\mathrm{nc})$ and the bulk $\mathrm{Si}$. From this, we get a value of $-0.4 \mathrm{eV}$ for the relaxation effect, and by use of the $-0.2 \mathrm{eV}$ binding energy difference, we obtain a chemical shift of $\Delta \epsilon=-0.6 \pm 0.1 \mathrm{eV}$ for the $\mathrm{Si}(\mathrm{nc})$ with respect to bulk $\mathrm{Si}$. This shift is also in the opposite direction of the expected quantum-size effect. ${ }^{22,35-36}$

To induce further charging/neutralization between the $\mathrm{Si}(\mathrm{nc})$ and the oxide matrix, and to get additional experimental evidence for differential charging between the two, we recorded the XPS spectra under external voltage stimuli. In Figure 4 we show the Si2p region of the sample annealed at $1100{ }^{\circ} \mathrm{C}$ recorded: (i) grounded, (ii) under $+10 \mathrm{~V}$, and (ii) $-10 \mathrm{~V}$ external bias, together with a silicon sample containing ca. a 6-nm thermal oxide layer for comparison. A small gold metal strip is attached to both samples for referencing. As we reported earlier for the silicon sample with a thin thermal oxide layer, the observed binding energy corresponding to the oxide layer $\left(\mathrm{Si}^{4+}\right)$ is decreased under positive bias (to $3.9 \pm 0.1 \mathrm{eV}$ ) and increased under negative bias (to $4.7 \pm 0.1 \mathrm{eV}$ ) due to reduced

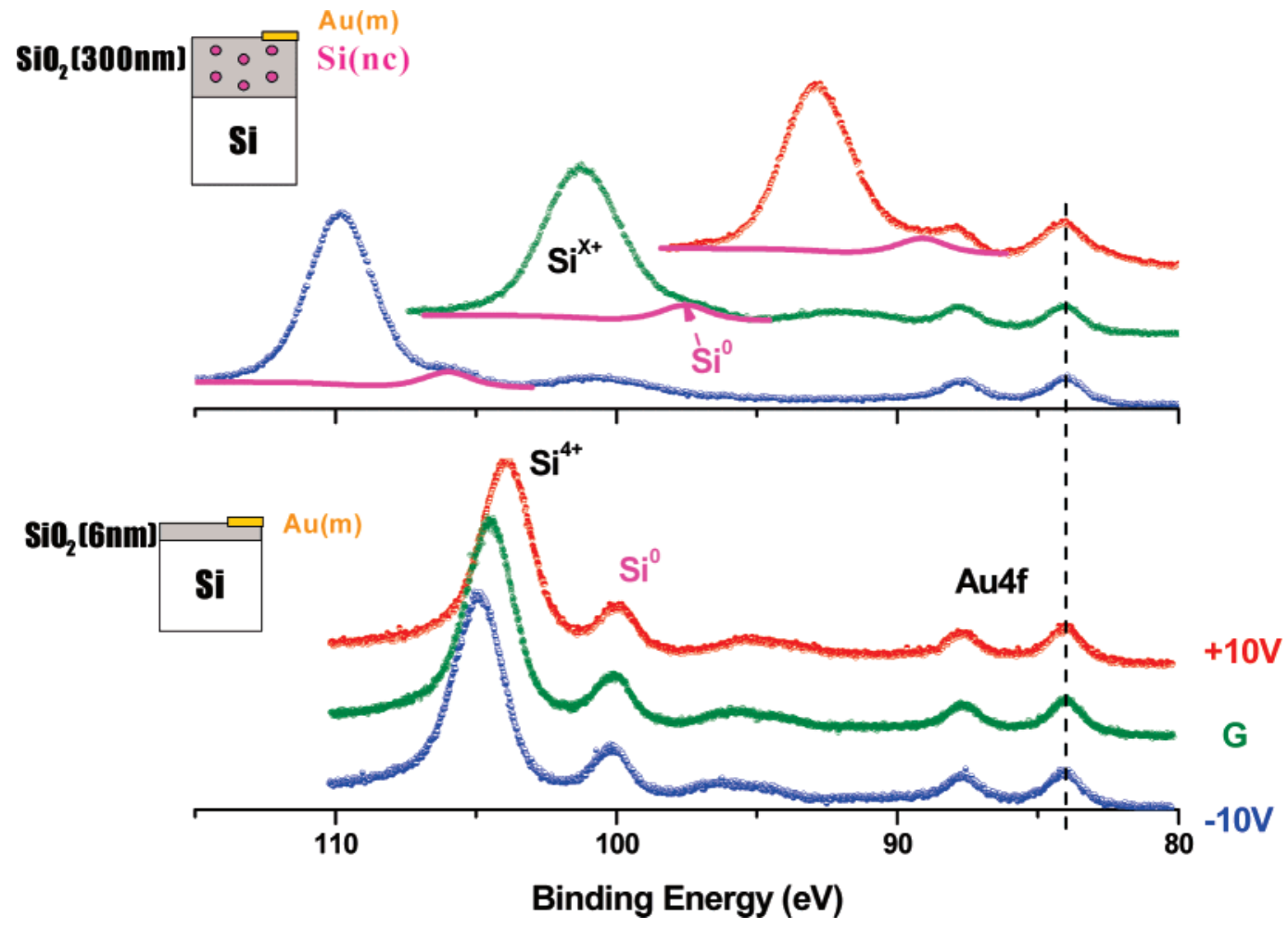

Figure 4. The $\mathrm{Si} 2 \mathrm{p}$ and the Au4f region under $+10 \mathrm{~V}$, ground, and $-10 \mathrm{~V}$ dc bias for the $\mathrm{Si}(\mathrm{nc})$ sample and a silicon sample containing a ca. 6 $\mathrm{nm}$ thermal oxide layer included to demonstrate our method of detecting differential charging between the oxide layer and silicon substrate. 


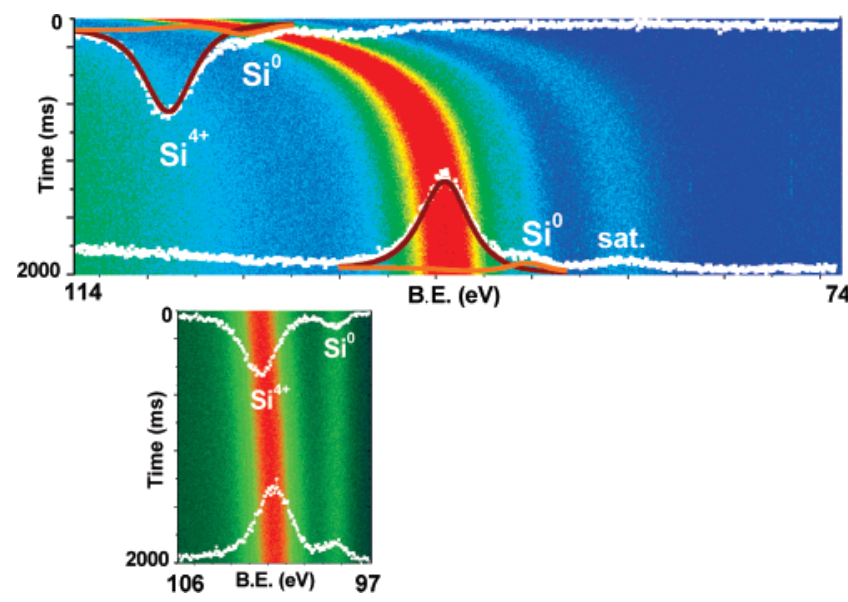

Figure 5. Two hundred time-resolved XPS spectra (with a 10-ms time resolution) of the samples in Figure 4.

(as a result of more effective neutralization by the slow electrons) and enhanced (as a result of less effective neutralization) charging of the layer, respectively. ${ }^{24,25}$ Even under +10 $\mathrm{V}$ bias we did not observe complete elimination of the charging of the thermal oxide layer. This is judged by the measured difference between the $\mathrm{Si} 2 \mathrm{p}\left(\mathrm{Si}^{4+}\right)$ and the $\mathrm{Au} 4 \mathrm{f}_{7 / 2}$ peaks to be larger than the theoretical value of $19.4 \mathrm{eV} .{ }^{26}$ When we examine the oxide sample containing $\mathrm{Si}(\mathrm{nc})$, we observe that the charging is much more severe, and the Si2p peaks shift more than $15 \mathrm{eV}$ when we change the bias from +10 and $-10 \mathrm{~V}$. This intense charging is related to the way the sample is prepared; PECVD produces many defects for trapping the charges induced by photoemission, as was also observed for the near stoichiometric $\mathrm{SiO}_{2}$ films. However, within our experimental capabilities, we were not able to induce additional differential charging (neither reduced nor enhanced) between the $\mathrm{Si}(\mathrm{nc})$ and the $\mathrm{SiO}_{2}$ host, as opposed to the case of a uniform 6-nm thermal oxide layer on silicon substrate.

We also performed time-resolved measurements of the same samples, as shown in Figure 5. These show 200 XPS spectra with a time-resolution of $10 \mathrm{~ms}$. As with the static measurements, the $\mathrm{Si} 2 \mathrm{p}\left(\mathrm{Si}^{4+}\right)$ of the sample with the 6 -nm thermal oxide undergoes a charging shift against the stable $\mathrm{Si}^{0}$ peak with a time constant of ca. $2 \mathrm{~s}^{29}$ In the case of the PECVD sample containing nanoclusters, both the $\mathrm{Si}^{4+}$ and the $\mathrm{Si}^{0}$ peaks undergo a large $(>15 \mathrm{eV})$ shift, with a similar time constant. This demonstrates once again that the PECVD silicon oxide layer and the silicon nanoclusters charge and discharge together, both in time and magnitude, under simultaneous X-ray and low energy electron irradiation, within our experimental limitations (10 ms time and $0.1 \mathrm{eV}$ energy resolution).

Recalling that Chen et al. ${ }^{15}$ reported a $-0.6 \mathrm{eV}$ shift, the same value as ours, without taking account of the matrix effect, using the measured binding energy difference from the full oxide, such a correction would put their value at $-1.0 \mathrm{eV}$, after including the $-0.4 \mathrm{eV}$ relaxation shift. Hence, by controlled charging/ discharging, we have obtained a measurement $(-0.6 \mathrm{eV})$ that is a step in the right direction. Still the very large magnitude of the absolute charging of the PECVD silicon oxide matrix does not allow us to completely eliminate differential charging between the $\mathrm{Si}(\mathrm{nc})$ and the $\mathrm{SiO}_{2}$ matrix (in order to correctly sort out the quantum-size effect of the silicon nanoclusters).

\section{Conclusions}

Although, a significant amount of binding energy shift due to charging can be induced, both in steady-state and in timeresolved fashions, in XPS peaks of the $\mathrm{Si}(\mathrm{nc})$ in the $\mathrm{SiO}_{2}$ matrix prepared by PECVD technique, surprisingly no measurable binding energy difference between the corresponding Si2p peaks could be detected. By use of the measured AP difference, we estimate a $-0.4-\mathrm{eV}$ shift for the relaxation energy, and combining it with the determined $4.3 \mathrm{eV}$ shift in the Si2p binding energy difference between the $\mathrm{Si}(\mathrm{nc})$ in the slicon oxide matrix, we obtain a shift of $-0.6 \mathrm{eV}$ for the $\mathrm{Si}(\mathrm{nc})$ from that of the bulk $\mathrm{Si}$, in the opposite direction of the expected quantum-size shift. Hence as long as one uses the oxide peak as the reference to determine the binding energy of the silicon clusters, differential charging is still the greatest obstacle to extract sizedependent binding energy shifts using XPS.

Acknowledgment. This work is partially supported by TUBA (Turkish Academy of Sciences) and TUBITAK (The Scientific and Technical Research Council of Turkey) through grants TBAG-2261 and TBAG-103T115.

\section{References and Notes}

(1) Canham, L. T. Appl. Phys. Lett. 1990, 57, 1046.

(2) Takagi, H.; Ogawa, H.; Yamazaki, Y.; Ishizaki, A.; Nakagiri, Appl. Phys. Lett. 1990, 56, 379.

(3) Cooke, D. W.; Bennett, B. L.; Frnum, E. H.; Hults, W. L.; Sickafus, K. E.; Smith, J. F.; Smith, J. L.; Taylor, T. N.; Tiwari, P. Appl. Phys. Lett. 1996, 68, 1663.

(4) Song, H. Z.; Bao, X. M. Phys. Rev. B 1997, 55, 6988.

(5) Ledoux, G.; Guillois, O.; Porterat, D.; Reynaud, C.; Huisken, F.; Kohn, B.; Paillard, V. Phys. Rev. B 2000, 62, 15942.

(6) Boer, E.; Brongersma, M. L.; Atwater, H. A.; Flagan, R. C.; Bell, L. D. Appl. Phys. Lett. 2001, 79, 791.

(7) Pavesi, 1.; Dal Negro, L.; Mazzoleni, C.; Franzo, G.; Priolo, F. Nature 2000, 408, 440.

(8) Kabashin, A. V.; Meunier, M. Appl. Phys. Lett. 2003, 82, 1619.

(9) Tiwari, S.; Rana, F.; Hanafi, H. I.; Hartstein, A.; Crabbe, E. F.; Chan, K. Appl. Phys. Lett. 1996, 68, 1377.

(10) Lin, G. R.; Lin, C. J.; Lin, C. K.; Chou, L. J.; Chueh, Y. L. J. Appl. Phys. 2005, 97, 94306.

(11) Kopanski, J. J.; Marchiando, J. F.; Lowney, J. R. J. Vac. Sci. Technol. B 1996, 14, 242.

(12) Iacona, F.; Lambardo, S.; Campisano, S. U. J. Vac. Sci. Technol. B 1996, 14, 2693.

(13) Chen, T. P.; Liu, Y.; Tse, M. S.; Tan, O. K.; Ho, P. F.; Liu, K. Y.; Gui, D.; Tan, A. L. K. Phys. Rev. B 2003, 68, 153301.

(14) Liu, Y.; Chen, T. P.; Fu, Y. Q.; Hsieh, J. H.; Ho, P. F.; Liu, Y. C. J. Phys. D: Appl. Phys. 2003, 36, L97.

(15) Liu, Y.; Fu, Y. Q.; Chen, T. P.; tse, M. S.; Fung, S.; Hsieh, J. H.; Yang, X. H. Jpn. J. Phys. 2003, 42, L1394.

(16) Ng, C. Y.; Chen, T. P.; Tse, M. S.; Lim, V. S. W.; Fung, S.; Tseng, A. Appl. Phys. Lett. 2005, 86, 152110.

(17) van Buuren, T.; Gao, Y.; Tiedje, T.; Dahn, J. R.; Way, B. M. Appl. Rev. Lett. 1992, 60, 3013.

(18) Dinh, L. N.; Chase, L. L.; Balooch, M.; Siekhaus, W. J.; Wooten,

F. Phys. Rev. B 1996, 54, 5029.

(19) van Buuren, T.; Dinh, L. N.; Chase, L. L.; Siekhaus, W. J.; Terminello, L. J. Phys. Rev. Lett. 1998, 80, 3803.

(20) Sun, C. Q.; Pan, L. K.; Fu, Y. Q.; Tay, B. K.; Li, S. J. Phys. Chem. $B$ 2003, 107, 5113 .

(21) Sun, C. Q.; Tay, B. K.; Fu, Y. Q.; Li, S.; Chen, T. P.; Bai, H. L.; Jiang, E. Y. J. Phys. Chem. B 2003, 107, 411.

(22) Iwata, S.; Ishizaka, A. J. Appl. Phys. 1996, 79, 6653.

(23) Chen, T. P.; Liu, Y.; Sun, C. Q.; Tse, M. S.; Hsieh, J. H.; Fu, Y.

Q.; Liu, Y. C.; Fung, S. J. J. Phys. Chem. B 2004, 108, 16609.

(24) Ulgut, B.; Suzer, S. J. Phys. Chem. B 2003, 107, 2939.

(25) Suzer, S. Anal. Chem. 2003, 75, 7026.

(26) Karadas, F.; Ertas, G.; Suzer, S. J. Phys. Chem. B 2004, 108, 1515.

(27) Demirok, U. K.; Ertas, G.; Suzer, S. J. Phys. Chem. B 2004, 108, 5179 .

(28) Ertas, G.; Suzer, S. Surf. Interface Anal. 2004, 36, 619

(29) Ertas, G.; Demirok, U. K.; Atalar, A.; Suzer, S. Appl. Phys. Lett. 2005, 86, 183110

(30) Wagner, C. D. Faraday Discuss. Chem. Soc. 1975, 60, 291.

(31) Kwok, R. W. M. (www.phy.cuhk.edu.hk/ surface/XPSPEAK/).

(32) Thomas, T. D.; Weightman, P. Phys. Rev. B 1986, 33, 5406.

(33) Mejias, J. A.; Jimenez, V. M.; Lassaletta, G.; Fernandez, A.;

Espinos, J. P.; Gonzalez- Elippe, A. R. J. Phys. Chem. 1996, 100, 16255.

(34) Leinen, D. Surf. Interface Anal. 2004, 36, 925.

(35) Yang, D. Q.; Sacher, E. Appl. Surf. Sci. 2002, $195,187$.

(36) Ohgi, T.; Fujita, D. Phys. Rev. B 2002, 66, 115410. 